\title{
Some results on fixed points of asymptotically strict quasi- $\phi$-pseudocontractions in the intermediate sense
}

\author{
Xiaolong Qin', Lin Wang ${ }^{2 *}$ and Shin Min Kang ${ }^{3}$
}

\section{"Correspondence:}

w164mail@yahoo.com.cn

${ }^{2}$ College of Statistics and

Mathematics, Yunnan University of

Finance and Economics, Kunming, 650221, China

Full list of author information is

available at the end of the article

\begin{abstract}
In this paper, a new nonlinear mapping, asymptotically strict quasi- $\phi$-pseudocontraction in the intermediate sense, is introduced. Projection algorithms are considered for the mapping. Strong convergence theorems for fixed points of the mapping are established based on projection algorithms in a real Banach space.

MSC: 47H09; 47J05; 47J25

Keywords: asymptotically quasi- $\phi$-nonexpansive mapping in the intermediate sense; asymptotically strict quasi- $\phi$-pseudocontraction; asymptotically strict quasi- $\phi$-pseudocontraction in the intermediate sense; fixed point
\end{abstract}

\section{Introduction}

Fixed point theory as an important branch of nonlinear analysis theory has been applied in the study of nonlinear phenomena. The theory itself is a beautiful mixture of analysis, topology, and geometry. Lots of problems arising in economics, engineering, and physics can be studied by fixed point techniques. The study of fixed point approximation algorithms for computing fixed points is now a topic of intensive research efforts. Many wellknown problems can be studied by using algorithms which are iterative in their nature. As an example, in computer tomography with limited data, each piece of information implies the existence of a convex set $C_{m}$ in which the required solution lies. The problem of finding a point in the intersection $\bigcap_{m=1}^{N} C_{m}$, where $N \geq 1$ is some positive integer, is then of crucial interest, and it cannot be usually solved directly. Therefore, an iterative algorithm must be used to approximate such point. The well-known convex feasibility problem, which captures applications in various disciplines such as image restoration and radiation therapy treatment planning, is to find a point in the intersection of common fixed point sets of a family of nonlinear mappings, see, for example, [1-6].

For iterative algorithms, the oldest and simplest one is the Picard iterative algorithm. It is known that $T$, where $T$ stands for a contractive mapping, enjoys a unique fixed point, and the sequence generated by the Picard iterative algorithm can converge to the unique fixed point. However, for more general nonexpansive mappings, the Picard iterative algorithm fails to converge to fixed points of nonexpansive mappings even when they enjoy fixed points. The Krasnoselskii-Mann iterative algorithm (one-step iterative algorithm) and the Ishikawa iterative algorithms (two-step iterative algorithm) have been studied for approx- 
imating fixed points of nonexpansive mappings and their extensions. However, both the Krasnoselskii-Mann iterative algorithm and the Ishikawa iterative algorithms are weak convergence for nonexpansive mappings only; see [7] and [8] for the classic weak convergence theorems. In many disciplines, including economics [9], image recovery [10], quantum physics [11], and control theory [12], problems arise in infinite dimension spaces. In such problems, strong convergence (norm convergence) is often much more desirable than weak convergence, for it translates the physically tangible property so that the energy $\left\|x_{n}-x\right\|$ of the error between the iterate $x_{n}$ and the solution $x$ eventually becomes arbitrarily small. The importance of strong convergence is also underlined in [13], where a convex function $f$ is minimized via the proximal-point algorithm: it is shown that the rate of convergence of the value sequence $\left\{f\left(x_{n}\right)\right\}$ is better when $\left\{x_{n}\right\}$ converges strongly than when it converges weakly. Such properties have a direct impact when the process is executed directly in the underlying infinite dimensional space. Projection methods, which were first introduced by Haugazeau [14], have been considered for the approximation of fixed points of nonexpansive mappings and their extensions. The advantage of projection methods is that strong convergence of iterative sequences can be guaranteed without any compact assumptions.

In this paper, a new class of new nonlinear mappings is introduced and studied. Based on a simple hybrid projection algorithm, a theorem of strong convergence for common fixed points is obtained. The results presented in this paper mainly improve the known corresponding results announced in the literature sources listed in this work.

The organization of this paper is as follows. In Section 2, we provide some necessary preliminaries. In Section 3, the hybrid projection algorithm is proposed and analyzed. With the help of the generalized projections, theorems of strong convergence are established. Some subresults of the main results are discussed.

\section{Preliminaries}

Let $H$ be a real Hilbert space, $C$ a nonempty subset of $H$, and $T: C \rightarrow C$ a nonlinear mapping. The symbol $F(T)$ stands for the fixed point set of $T$. Recall the following. $T$ is said to be nonexpansive if

$$
\|T x-T y\| \leq\|x-y\|, \quad \forall x, y \in C .
$$

$T$ is said to be quasi-nonexpansive if $F(T) \neq \emptyset$ and

$$
\|p-T y\| \leq\|p-y\|, \quad \forall p \in F(T), \forall y \in C .
$$

$T$ is said to be asymptotically nonexpansive if there exists a sequence $\left\{\mu_{n}\right\} \subset[0, \infty)$ with $\mu_{n} \rightarrow 0$ as $n \rightarrow \infty$ such that

$$
\left\|T^{n} x-T^{n} y\right\| \leq\left(1+\mu_{n}\right)\|x-y\|, \quad \forall x, y \in C, \forall n \geq 1 .
$$

The class of asymptotically nonexpansive mappings was introduced by Goebel and Kirk [15]. Since 1972, a host of authors have studied the convergence of iterative algorithms for such a class of mappings. 
$T$ is said to be asymptotically quasi-nonexpansive if $F(T) \neq \emptyset$ and there exists a sequence $\left\{\mu_{n}\right\} \subset[0, \infty)$ with $\mu_{n} \rightarrow 0$ as $n \rightarrow \infty$ such that

$$
\left\|p-T^{n} y\right\| \leq\left(1+\mu_{n}\right)\|p-y\|, \quad \forall p \in F(T), \forall y \in C, \forall n \geq 1 .
$$

$T$ is said to be asymptotically nonexpansive in the intermediate sense if it is continuous and the following inequality holds:

$$
\limsup _{n \rightarrow \infty} \sup _{x, y \in C}\left(\left\|T^{n} x-T^{n} y\right\|-\|x-y\|\right) \leq 0 .
$$

If we define

$$
\xi_{n}=\max \left\{0, \sup _{x, y \in C}\left(\left\|T^{n} x-T^{n} y\right\|-\|x-y\|\right)\right\},
$$

then $\xi_{n} \rightarrow 0$ as $n \rightarrow \infty$. It follows that (2.1) is reduced to

$$
\left\|T^{n} x-T^{n} y\right\| \leq\|x-y\|+\xi_{n}, \quad \forall x, y \in C, \forall n \geq 1 .
$$

The class of mappings which are asymptotically nonexpansive in the intermediate sense was considered by Bruck, Kuczumow, and Reich [16]. It is worth mentioning that the class of mappings which are asymptotically nonexpansive in the intermediate sense may not be Lipschitz continuous; see [16-18].

$T$ is said to be asymptotically quasi-nonexpansive in the intermediate sense if $F(T) \neq \varnothing$ and the following inequality holds:

$$
\limsup _{n \rightarrow \infty} \sup _{p \in F(T), y \in C}\left(\left\|p-T^{n} y\right\|-\|p-y\|\right) \leq 0 .
$$

If we define

$$
\xi_{n}=\max \left\{0, \sup _{p \in F(T), y \in C}\left(\left\|p-T^{n} y\right\|-\|p-y\|\right)\right\},
$$

then $\xi_{n} \rightarrow 0$ as $n \rightarrow \infty$. It follows that (2.2) is reduced to

$$
\left\|p-T^{n} y\right\| \leq\|p-y\|+\xi_{n}, \quad \forall p \in F(T), \forall y \in C, \forall n \geq 1 .
$$

$T$ is said to be strictly pseudocontractive if there exists a constant $\kappa \in[0,1)$ such that

$$
\|T x-T y\|^{2} \leq\|x-y\|^{2}+\kappa\|(I-T) x-(I-T) y\|^{2}, \quad \forall x, y \in C .
$$

The class of strictly pseudocontractive mappings was introduced by Browder and Petryshyn [19]. It is easy to see that the class of strictly pseudocontractive mappings includes the class of nonexpansive mappings as a special case. In 2007, Marino and Xu [20] proved that the fixed point set of strict pseudocontractions is closed and convex. They also obtained a strong convergence theorem for fixed points of the class of mappings based on hybrid projection algorithms; see [20] for more details. 
$T$ is said to be strictly quasi-pseudocontractive if $F(T) \neq \emptyset$ and there exists a constant $\kappa \in[0,1)$ such that

$$
\|p-T y\|^{2} \leq\|p-y\|^{2}+\kappa\|y-T y\|^{2}, \quad \forall p \in F(T), \forall y \in C .
$$

$T$ is said to be an asymptotically strict pseudocontraction if there exist a constant $\kappa \in$ $[0,1)$ and a sequence $\left\{\mu_{n}\right\} \subset[0, \infty)$ with $\mu_{n} \rightarrow 0$ as $n \rightarrow \infty$ such that

$$
\left\|T^{n} x-T^{n} y\right\|^{2} \leq\left(1+\mu_{n}\right)\|x-y\|^{2}+\kappa\left\|\left(I-T^{n}\right) x-\left(I-T^{n}\right) y\right\|^{2}, \quad \forall x, y \in C, \forall n \geq 1 .
$$

The class of asymptotically strict pseudocontractions was introduced by Qihou [21] in 1996. Kim and Xu [22] proved that the fixed point set of asymptotically strict pseudocontractions is closed and convex. They also obtained a strong convergence theorem for fixed points of the class of asymptotically strict pseudocontractions based on projection algorithms; see [22] for more details.

$T$ is said to be an asymptotically strict quasi-pseudocontraction if there exist a constant $\kappa \in[0,1), F(T) \neq \emptyset$ and a sequence $\left\{\mu_{n}\right\} \subset[0, \infty)$ with $\mu_{n} \rightarrow 0$ as $n \rightarrow \infty$ such that

$$
\left\|p-T^{n} y\right\|^{2} \leq\left(1+\mu_{n}\right)\|p-y\|^{2}+\kappa\left\|y-T^{n} y\right\|^{2}, \quad \forall p \in F(T), y \in C, \forall n \geq 1 .
$$

$T$ is said to be an asymptotically strict pseudocontraction in the intermediate sense if there exist a constant $\kappa \in[0,1)$ and a sequence $\left\{\mu_{n}\right\} \subset[0, \infty)$ with $\mu_{n} \rightarrow 0$ as $n \rightarrow \infty$ such that

$$
\limsup _{n \rightarrow \infty} \sup _{x, y \in C}\left(\left\|T^{n} x-T^{n} y\right\|^{2}-\left(1+\mu_{n}\right)\|x-y\|^{2}-\kappa\left\|\left(I-T^{n}\right) x-\left(I-T^{n}\right) y\right\|^{2}\right) \leq 0 .
$$

Put

$$
\xi_{n}=\max \left\{0, \sup _{x, y \in C}\left(\left\|T^{n} x-T^{n} y\right\|^{2}-\left(1+\mu_{n}\right)\|x-y\|^{2}-\kappa\left\|\left(I-T^{n}\right) x-\left(I-T^{n}\right) y\right\|^{2}\right)\right\} .
$$

It follows that $\xi_{n} \rightarrow 0$ as $n \rightarrow \infty$. Then, (2.3) is reduced to the following:

$$
\begin{aligned}
\left\|T^{n} x-T^{n} y\right\|^{2} \leq & \left(1+\mu_{n}\right)\|x-y\|^{2} \\
& +\kappa\left\|\left(I-T^{n}\right) x-\left(I-T^{n}\right) y\right\|^{2}+\xi_{n}, \quad \forall x \in F(T), \forall y \in C, \forall n \geq 1 .
\end{aligned}
$$

The class of mappings was introduced by Sahu, $\mathrm{Xu}$, and Yao [23]. They proved that the fixed point set of asymptotically strict pseudocontractions in the intermediate sense is closed and convex. They also obtained a strong convergence theorem for fixed points of the class of mappings based on projection algorithms; see [23] for more details.

$T$ is said to be an asymptotically strict quasi-pseudocontraction in the intermediate sense if there exist a constant $\kappa \in[0,1), F(T) \neq \emptyset$ and a sequence $\left\{\mu_{n}\right\} \subset[0, \infty)$ with $\mu_{n} \rightarrow 0$ as $n \rightarrow \infty$ such that

$$
\limsup _{n \rightarrow \infty} \sup _{p \in F(T), y \in C}\left(\left\|p-T^{n} y\right\|^{2}-\left(1+\mu_{n}\right)\|p-y\|^{2}-\kappa\left\|y-T^{n} y\right\|^{2}\right) \leq 0 .
$$


Put

$$
\xi_{n}=\max \left\{0, \sup _{p \in F(T), y \in C}\left(\left\|p-T^{n} y\right\|^{2}-\left(1+\mu_{n}\right)\|p-y\|^{2}-\kappa\left\|y-T^{n} y\right\|^{2}\right)\right\} .
$$

It follows that $\xi_{n} \rightarrow 0$ as $n \rightarrow \infty$. Then, (2.4) is reduced to the following:

$$
\left\|p-T^{n} y\right\|^{2} \leq\left(1+\mu_{n}\right)\|p-y\|^{2}+\kappa\left\|y-T^{n} y\right\|^{2}+\xi_{n}, \quad \forall p \in F(T), \forall y \in C, \forall n \geq 1 .
$$

In what follows, we always assume that $E$ is a Banach space with the dual space $E^{*}$. The symbol $J$ stands for the normalized duality mapping from $E$ to $2^{E^{*}}$ defined by

$$
J x=\left\{f^{*} \in E^{*}:\left\langle x, f^{*}\right\rangle=\|x\|^{2}=\left\|f^{*}\right\|^{2}\right\}, \quad \forall x \in E,
$$

where $\langle\cdot, \cdot\rangle$ denotes the generalized duality pairing of elements between $E$ and $E^{*}$. It is well known that if $E^{*}$ is strictly convex, then $J$ is single valued; if $E^{*}$ is uniformly convex, then $J$ is uniformly continuous on bounded subsets of $E$; if $E^{*}$ is reflexive and smooth, then $J$ is single valued and demicontinuous.

It is also well known that if $C$ is a nonempty closed convex subset of a Hilbert space $H$ and $P_{C}: H \rightarrow C$ is the metric projection of $H$ onto $C$, then $P_{C}$ is nonexpansive. This fact actually characterizes Hilbert spaces, and consequently, it is not available in more general Banach spaces. In this connection, Alber [24] introduced a generalized projection operator $\Pi_{C}$ in a Banach space $E$ which is an analogue of the metric projection in Hilbert spaces.

Recall that a Banach space $E$ is said to be strictly convex if $\left\|\frac{x+y}{2}\right\|<1$ for all $x, y \in E$ with $\|x\|=\|y\|=1$ and $x \neq y$. It is said to be uniformly convex if $\lim _{n \rightarrow \infty}\left\|x_{n}-y_{n}\right\|=0$ for any two sequences $\left\{x_{n}\right\}$ and $\left\{y_{n}\right\}$ in $E$ such that $\left\|x_{n}\right\|=\left\|y_{n}\right\|=1$ and $\lim _{n \rightarrow \infty}\left\|\frac{x_{n}+y_{n}}{2}\right\|=1$. Let $U_{E}=\{x \in E:\|x\|=1\}$ be the unit sphere of $E$. Then the Banach space $E$ is said to be smooth provided $\lim _{t \rightarrow 0} \frac{\|x+t y\|-\|x\|}{t}$ exists for all $x, y \in U_{E}$. It is also said to be uniformly smooth if the limit is attained uniformly for all $x, y \in U_{E}$.

Recall that a Banach space $E$ has the Kadec-Klee property if for any sequence $\left\{x_{n}\right\} \subset E$ and $x \in E$ with $x_{n} \rightarrow x$ and $\left\|x_{n}\right\| \rightarrow\|x\|$, then $\left\|x_{n}-x\right\| \rightarrow 0$ as $n \rightarrow \infty$. For more details on the Kadec-Klee property, the readers can refer to [25-27] and the references therein. It is well known that if $E$ is a uniformly convex Banach space, then $E$ satisfies the Kadec-Klee property.

Let $E$ be a smooth Banach space. Consider the functional defined by

$$
\phi(x, y)=\|x\|^{2}-2\langle x, J y\rangle+\|y\|^{2}, \quad \forall x, y \in E .
$$

Notice that, in a Hilbert space $H$, (2.5) is reduced to $\phi(x, y)=\|x-y\|^{2}$ for all $x, y \in H$. The generalized projection $\Pi_{C}: E \rightarrow C$ is a mapping that assigns to an arbitrary point $x \in E$, the minimum point of the functional $\phi(x, y)$; that is, $\Pi_{C} x=\bar{x}$, where $\bar{x}$ is the solution to the following minimization problem:

$$
\phi(\bar{x}, x)=\min _{y \in C} \phi(y, x) .
$$


The existence and uniqueness of the operator $\Pi_{C}$ follow from the properties of the functional $\phi(x, y)$ and the strict monotonicity of the mapping $J$; see, for example, [24-27]. In Hilbert spaces, $\Pi_{C}=P_{C}$. It is obvious from the definition of the function $\phi$ that

$$
(\|y\|-\|x\|)^{2} \leq \phi(y, x) \leq(\|y\|+\|x\|)^{2}, \quad \forall x, y \in E,
$$

and

$$
\phi(x, y)=\phi(x, z)+\phi(z, y)+2\langle x-z, J z-J y\rangle, \quad \forall x, y, z \in E .
$$

Remark 2.1 If $E$ is a reflexive, strictly convex, and smooth Banach space, then for all $x, y \in$ $E, \phi(x, y)=0$ if and only if $x=y$. It is sufficient to show that if $\phi(x, y)=0$, then $x=y$. From (2.6), we have $\|x\|=\|y\|$. This implies that $\langle x, J y\rangle=\|x\|^{2}=\|J y\|^{2}$. From the definition of $J$, we see that $J x=J y$. It follows that $x=y$; see $[25,27]$ for more details.

Next, we recall the following.

(1) A point $p$ in $C$ is said to be an asymptotic fixed point of $T$ [28] if $C$ contains a sequence $\left\{x_{n}\right\}$ which converges weakly to $p$ such that $\lim _{n \rightarrow \infty}\left\|x_{n}-T x_{n}\right\|=0$. The set of asymptotic fixed points of $T$ will be denoted by $\widetilde{F}(T)$.

(2) $T$ is said to be relatively nonexpansive if

$$
\widetilde{F}(T)=F(T) \neq \emptyset, \quad \phi(p, T x) \leq \phi(p, x), \quad \forall x \in C, \forall p \in F(T) .
$$

The asymptotic behavior of relatively nonexpansive mappings was studied in [29-31].

(3) $T$ is said to be relatively asymptotically nonexpansive if

$$
\widetilde{F}(T)=F(T) \neq \emptyset, \quad \phi\left(p, T^{n} x\right) \leq\left(1+\mu_{n}\right) \phi(p, x), \quad \forall x \in C, \forall p \in F(T), \forall n \geq 1,
$$

where $\left\{\mu_{n}\right\} \subset[0, \infty)$ is a sequence such that $\mu_{n} \rightarrow 1$ as $n \rightarrow \infty$.

Remark 2.2 The class of relatively asymptotically nonexpansive mappings was first considered in Su and Qin [32]; see also, Agarwal, Cho, and Qin [33], and Qin et al. [34].

(4) $T$ is said to be quasi- $\phi$-nonexpansive if

$$
F(T) \neq \emptyset, \quad \phi(p, T x) \leq \phi(p, x), \quad \forall x \in C, \forall p \in F(T) .
$$

(5) $T$ is said to be asymptotically quasi- $\phi$-nonexpansive if there exists a sequence $\left\{\mu_{n}\right\} \subset$ $[0, \infty)$ with $\mu_{n} \rightarrow 0$ as $n \rightarrow \infty$ such that

$$
F(T) \neq \emptyset, \quad \phi\left(p, T^{n} x\right) \leq\left(1+\mu_{n}\right) \phi(p, x), \quad \forall x \in C, \forall p \in F(T), \forall n \geq 1 .
$$

Remark 2.3 The class of quasi- $\phi$-nonexpansive mappings and the class of asymptotically quasi- $\phi$-nonexpansive mappings were considered in Qin, Cho, and Kang [35], and Zhou, Gao, and Tan [36]; see also [37-42]. 
Remark 2.4 The class of quasi- $\phi$-nonexpansive mappings and the class of asymptotically quasi- $\phi$-nonexpansive mappings are more general than the class of relatively nonexpansive mappings and the class of relatively asymptotically nonexpansive mappings. Quasi$\phi$-nonexpansive mappings and asymptotically quasi- $\phi$-nonexpansive mappings do not require $F(T)=\widetilde{F}(T)$.

Remark 2.5 The class of quasi- $\phi$-nonexpansive mappings and the class of asymptotically quasi- $\phi$-nonexpansive mappings are generalizations of the class of quasi-nonexpansive mappings and the class of asymptotically quasi-nonexpansive mappings in Banach spaces.

(6) $T$ is said to be a strict quasi- $\phi$-pseudocontraction if $F(T) \neq \varnothing$ and there exists a constant $\kappa \in[0,1)$ such that

$$
\phi(p, T x) \leq \phi(p, x)+\kappa \phi(x, T x), \quad \forall x \in C, p \in F(T) .
$$

(7) $T$ is said to be an asymptotically strict quasi- $\phi$-pseudocontraction if $F(T) \neq \emptyset$ and there exists a sequence $\left\{\mu_{n}\right\} \subset[0, \infty)$ with $\mu_{n} \rightarrow 0$ as $n \rightarrow \infty$ and a constant $\kappa \in[0,1)$ such that

$$
\phi\left(p, T^{n} x\right) \leq\left(1+\mu_{n}\right) \phi(p, x)+\kappa \phi\left(x, T^{n} x\right), \quad \forall x \in C, \forall p \in F(T), \forall n \geq 1 .
$$

Remark 2.6 It is clear that strict quasi- $\phi$-pseudocontractions are asymptotically strict quasi- $\phi$-pseudocontractions with the sequence $\mu_{n} \equiv 0$. The class of asymptotically strict quasi- $\phi$-pseudocontractions was first considered in Qin et al. [43]; see [43] for more details on asymptotically strict quasi- $\phi$-pseudocontractions and see [44] for more details on quasi-strict pseudocontractions and the references therein.

Remark 2.7 If $\kappa=0$, then the class of asymptotically strict quasi- $\phi$-pseudocontractions is reduced to asymptotically quasi- $\phi$-nonexpansive mappings.

Remark 2.8 The class of strict quasi- $\phi$-pseudocontraction and the class of asymptotically strict quasi- $\phi$-pseudocontractions are generalizations of the class of strict quasipseudocontraction and the class of asymptotically strict quasi-pseudocontractions in Banach spaces.

(8) The mapping $T$ is said to be asymptotically regular on $C$ if, for any bounded subset $K$ of $C$,

$$
\lim _{n \rightarrow \infty} \sup _{x \in K}\left\{\left\|T^{n+1} x-T^{n} x\right\|\right\}=0 .
$$

In this paper, based on the class of asymptotically strict pseudocontractions in the intermediate sense which was introduced by Sahu, $\mathrm{Xu}$, and Yao [23] in Hilbert spaces, we introduce and consider the following new nonlinear mapping: asymptotically strict quasi$\phi$-pseudocontraction in the intermediate sense.

(9) $T$ is said to be an asymptotically strict quasi- $\phi$-pseudocontraction in the intermediate sense if $F(T) \neq \emptyset$ and there exists a sequence $\left\{\mu_{n}\right\} \subset[0, \infty)$ with $\mu_{n} \rightarrow 0$ as $n \rightarrow \infty$ and a 
constant $\kappa \in[0,1)$ such that

$$
\limsup _{n \rightarrow \infty} \sup _{p \in F(T), x \in C}\left(\phi\left(p, T^{n} x\right)-\left(1+\mu_{n}\right) \phi(p, x)-\kappa \phi\left(x, T^{n} x\right)\right) \leq 0 .
$$

Put

$$
\xi_{n}=\max \left\{0, \sup _{p \in F(T), x \in C}\left(\phi\left(p, T^{n} x\right)-\left(1+\mu_{n}\right) \phi(p, x)-\kappa \phi\left(x, T^{n} x\right)\right)\right\} .
$$

It follows that $\xi_{n} \rightarrow 0$ as $n \rightarrow \infty$. Then, (2.8) is reduced to the following:

$$
\phi\left(p, T^{n} x\right) \leq\left(1+\mu_{n}\right) \phi(p, x)+\kappa \phi\left(x, T^{n} x\right)+\xi_{n}, \quad \forall p \in F(T), \forall x \in C, \forall n \geq 1 .
$$

Remark 2.9 The class of asymptotically strict quasi- $\phi$-pseudocontractions in the intermediate sense is a generalization of the class of asymptotically strict quasi-pseudocontractions in the intermediate sense in the framework of Banach spaces. For examples of the mapping in $\mathbb{R}^{1}$, we refer the readers to Sahu, Xu, and Yao [23].

Remark 2.10 If $\kappa=0$ and $\mu_{n} \equiv 0$, then we call $T$ an asymptotically quasi- $\phi$-nonexpansive mapping in the intermediate sense.

Remark 2.11 The class of asymptotically quasi- $\phi$-nonexpansive mappings in the intermediate sense is a generalization of the class of asymptotically quasi-nonexpansive mappings in the intermediate sense in the framework of Banach spaces.

In order to prove our main results, we also need the following lemmas:

Lemma 2.12 [45] Let E be a uniformly convex and smooth Banach space. Let $\left\{x_{n}\right\}$ and $\left\{y_{n}\right\}$ be two sequences in E. If $\phi\left(x_{n}, y_{n}\right) \rightarrow 0$ and either $\left\{x_{n}\right\}$ or $\left\{y_{n}\right\}$ is bounded, then $x_{n}-y_{n} \rightarrow 0$ as $n \rightarrow \infty$.

Lemma 2.13 [24] Let $C$ be a nonempty, closed, and convex subset of a smooth Banach space $E$, and $x \in E$. Then $x_{0}=\Pi_{C} x$ if and only if

$$
\left\langle x_{0}-y, J x-J x_{0}\right\rangle \geq 0, \quad \forall y \in C
$$

Lemma 2.14 [24] Let $E$ be a reflexive, strictly convex, and smooth Banach space, $C$ a nonempty, closed, and convex subset of $E$, and $x \in E$. Then

$$
\phi\left(y, \Pi_{C} x\right)+\phi\left(\Pi_{C} x, x\right) \leq \phi(y, x), \quad \forall y \in C .
$$

\section{Main results}

Now, we are in a position to give the main results in this paper.

Theorem 3.1 Let E be a uniformly convex and smooth Banach space. Let $C$ be a nonempty closed and convex subset of $E$. Let $\Lambda$ be an index set and $T_{i}: C \rightarrow C$, where $i \in \Lambda$, be an asymptotically strict quasi- $\phi$-pseudocontraction in the intermediate sense with a sequence 
$\left\{\mu_{(n, i)}\right\} \subset[0, \infty)$ such that $\mu_{(n, i)} \rightarrow 0$ as $n \rightarrow \infty$. Assume that $\bigcap_{i \in \Lambda} F\left(T_{i}\right) \neq \emptyset$. For each $i \in \Lambda$, assume that $T_{i}$ is closed and uniformly asymptotically regular on $C$, and $F\left(T_{i}\right)$ is bounded. Let $\left\{x_{n}\right\}$ be a sequence generated in the following manner:

$$
\left\{\begin{array}{l}
x_{0} \in E, \quad \text { chosen arbitrarily, } \\
C_{(1, i)}=C, \\
C_{1}=\bigcap_{i \in \Lambda} C_{(1, i)}, \\
x_{1}=\prod_{C_{1}} x_{0}, \\
C_{(n+1, i)}=\left\{u \in C_{(n, i)}:\right. \\
\left.\quad \phi\left(x_{n}, T_{i}^{n} x_{n}\right) \leq \frac{2}{1-\kappa_{i}}\left\langle x_{n}-u, J x_{n}-J T_{i}^{n} x_{n}\right\}+\mu_{(n, i)} \frac{M_{(n, i)}}{1-\kappa_{i}}+\frac{\xi(n, i)}{1-\kappa_{i}}\right\}, \\
C_{n+1}=\bigcap_{i \in \Lambda} C_{(n+1, i)}, \\
x_{n+1}=\prod_{C_{n+1}} x_{0}, \quad \forall n \geq 0,
\end{array}\right.
$$

where $M_{(n, i)}=\sup \left\{\phi\left(p, x_{n}\right): p \in F\left(T_{i}\right)\right\}$ and

$$
\xi_{(n, i)}=\max \left\{0, \sup _{p \in F\left(T_{i}\right), x \in C}\left(\phi\left(p, T_{i}^{n} x\right)-\left(1+\mu_{(n, i)}\right) \phi(p, x)-\kappa_{i} \phi\left(x, T_{i}^{n} x\right)\right)\right\} .
$$

Then the sequence $\left\{x_{n}\right\}$ converges strongly to $\bar{x}=\Pi_{\bigcap_{i \in \Lambda} F\left(T_{i}\right)} x_{0}$.

Proof First, we show, for every $i \in \Lambda$, that $F\left(T_{i}\right)$ is closed and convex. This proves that $\Pi_{\bigcap_{i \in \Lambda} F\left(T_{i}\right)} x_{0}$ is well defined for all $x_{0} \in E$. On the closedness of $F\left(T_{i}\right)$, we can easily draw the desired conclusion from the closedness of $T_{i}$. Now, we are in a position to show the convexness of $F\left(T_{i}\right), \forall i \in \Lambda$. Let $p_{(1, i)}, p_{(2, i)} \in F\left(T_{i}\right)$ and $p_{i}=t_{i} p_{(1, i)}+\left(1-t_{i}\right) p_{(2, i)}$, where $t_{i} \in$ $(0,1)$, for every $i \in \Lambda$. We see that $p_{i}=T_{i} p_{i}$. Indeed, we see from the definition of $T_{i}$ that

$$
\phi\left(p_{(1, i)}, T_{i}^{n} p_{i}\right) \leq\left(1+\mu_{(n, i)}\right) \phi\left(p_{(1, i)}, p_{i}\right)+\kappa_{i} \phi\left(p_{i}, T_{i}^{n} p_{i}\right)+\xi_{(n, i)},
$$

and

$$
\phi\left(p_{(2, i)}, T_{i}^{n} p_{i}\right) \leq\left(1+\mu_{(n, i)}\right) \phi\left(p_{(2, i)}, p_{i}\right)+\kappa_{i} \phi\left(p_{i}, T_{i}^{n} p_{i}\right)+\xi_{(n, i)} .
$$

It follows from (2.7) that

$$
\phi\left(p_{i}, T_{i}^{n} p_{i}\right) \leq \frac{\mu_{(n, i)}}{1-\kappa_{i}} \phi\left(p_{(1, i)}, p_{i}\right)+\frac{2}{1-\kappa_{i}}\left\langle p_{i}-p_{(1, i)}, J p_{i}-J T_{i}^{n} p_{i}\right\rangle+\frac{\xi_{(n, i)}}{1-\kappa_{i}},
$$

and

$$
\phi\left(p_{i}, T_{i}^{n} p_{i}\right) \leq \frac{\mu_{(n, i)}}{1-\kappa_{i}} \phi\left(p_{(2, i)}, p_{i}\right)+\frac{2}{1-\kappa_{i}}\left\langle p_{i}-p_{(2, i)}, J p_{i}-J T_{i}^{n} p_{i}\right\rangle+\frac{\xi_{(n, i)}}{1-\kappa_{i}} .
$$

Multiplying $t_{i}$ and $\left(1-t_{i}\right)$ on both sides of (3.1) and (3.2) respectively yields that

$$
\phi\left(p_{i}, T_{i}^{n} p_{i}\right) \leq \frac{t_{i} \mu_{(n, i)}}{1-\kappa_{i}} \phi\left(p_{(1, i)}, p_{i}\right)+\frac{\left(1-t_{i}\right) \mu_{(n, i)}}{1-\kappa_{i}} \phi\left(p_{(2, i)}, p_{i}\right)+\frac{\xi_{(n, i)}}{1-\kappa_{i}} .
$$


It follows that

$$
\lim _{n \rightarrow \infty} \phi\left(p_{i}, T_{i}^{n} p_{i}\right)=0
$$

In view of Lemma 2.12, we see that $T_{i}^{n} p_{i} \rightarrow p_{i}$ as $n \rightarrow \infty$ for each $i \in \Lambda$. This implies $T_{i} T_{i}^{n} p_{i}=T_{i}^{n+1} p_{i} \rightarrow p_{i}$ as $n \rightarrow \infty$ for each $i \in \Lambda$. From the closedness of $T_{i}$, we obtain $p_{i} \in F\left(T_{i}\right)$. This proves that $F\left(T_{i}\right)$ is convex. This completes the proof that $F\left(T_{i}\right)$ is closed and convex.

Next, we prove that $C_{n}$ is closed and convex for each $n \geq 1$. It suffices to show that, for each fixed but arbitrary $i \in \Lambda, C_{(n, i)}$ is closed and convex for each $n \geq 1$. This can be proved by induction on $n$. It is obvious that $C_{(1, i)}=C$ is closed and convex. Assume that $C_{(h, i)}$ is closed and convex for some $h \geq 1$. Let $a, b \in C_{(h+1, i)}$ and $c=t a+(1-t) b$, where $t \in(0,1)$. It follows that

$$
\phi\left(x_{h}, T_{i}^{h} x_{h}\right) \leq \frac{2}{1-\kappa_{i}}\left\langle x_{h}-a, J x_{h}-J T_{i}^{h} x_{h}\right\rangle+\mu_{(h, i)} \frac{M_{(h, i)}}{1-\kappa_{h}}+\frac{\xi_{(h, i)}}{1-\kappa_{i}},
$$

and

$$
\phi\left(x_{h}, T_{i}^{h} x_{h}\right) \leq \frac{2}{1-\kappa_{i}}\left\langle x_{h}-b, J x_{h}-J T_{i}^{h} x_{h}\right\rangle+\mu_{(h, i)} \frac{M_{(h, i)}}{1-\kappa_{i}}+\frac{\xi_{(h, i)}}{1-\kappa_{i}}
$$

where $a, b \in C_{(h, i)}$. From the above two inequalities, we find that

$$
\phi\left(x_{h}, T_{i}^{h} x_{h}\right) \leq \frac{2}{1-\kappa_{i}}\left\langle x_{h}-c, J x_{h}-J T_{i}^{h} x_{h}\right\rangle+\mu_{(h, i)} \frac{M_{(h, i)}}{1-\kappa_{i}}+\frac{\xi_{(h, i)}}{1-\kappa_{i}}
$$

where $c \in C_{(h, i)}$. It follows that $C_{(h+1, i)}$ is closed, and convex. This, in turn, implies that $C_{n}=\bigcap_{i \in \Lambda} C_{(n, i)}$ is closed and convex.

Now, we are in a position to show, for each $i \in \Lambda$, that $F\left(T_{i}\right) \subset C_{n}$. It is obvious that $F\left(T_{i}\right) \subset C=C_{1}$. Suppose that $F\left(T_{i}\right) \subset C_{h}$ for some $h \in \mathbb{N}$. For any $z \in F\left(T_{i}\right) \subset C_{h}$, we see that

$$
\phi\left(z, T_{i}^{h} x_{h}\right) \leq\left(1+\mu_{(h, i)}\right) \phi\left(z, x_{h}\right)+\kappa_{i} \phi\left(x_{h}, T_{i}^{h} x_{h}\right)+\xi_{(h, i)}
$$

On the other hand, we obtain from (2.7) that

$$
\phi\left(z, T_{i}^{h} x_{h}\right)=\phi\left(z, x_{h}\right)+\phi\left(x_{h}, T_{i}^{h} x_{h}\right)+2\left\langle z-x_{h}, J x_{h}-J T_{i}^{h} x_{h}\right\rangle .
$$

Combining (3.3) with (3.4), we arrive at

$$
\begin{aligned}
\phi\left(x_{h}, T_{i}^{h} x_{h}\right) & \leq \frac{\mu_{(h, i)}}{1-\kappa_{i}} \phi\left(z, x_{h}\right)+\frac{2}{1-\kappa_{i}}\left\langle x_{h}-z, J x_{h}-J T_{i}^{h} x_{h}\right\rangle+\frac{\xi_{(h, i)}}{1-\kappa_{i}} \\
& \leq \mu_{(h, i)} \frac{M_{(h, i)}}{1-\kappa_{i}}+\frac{2}{1-\kappa_{i}}\left\langle x_{h}-z, J x_{h}-J T_{i}^{h} x_{h}\right\rangle+\frac{\xi_{(h, i)}}{1-\kappa_{i}}
\end{aligned}
$$

which implies that $z \in C_{(h+1, i)}$. This proves, for each $i \in \Lambda$, that $F\left(T_{i}\right) \subset C_{n}$. This implies that $\bigcap_{i \in \Lambda} F\left(T_{i}\right) \subset C_{n}$. 
In view of $x_{n}=\Pi_{C_{n}} x_{0}$, we see from Lemma 2.13 that

$$
\left\langle x_{n}-z, J x_{0}-J x_{n}\right\rangle \geq 0, \quad \forall z \in C_{n} .
$$

This implies that

$$
\left\langle x_{n}-w, J x_{0}-J x_{n}\right\rangle \geq 0, \quad \forall w \in \bigcap_{i \in \Lambda} F\left(T_{i}\right) \subset C_{n} .
$$

It follows from Lemma 2.14 that

$$
\begin{aligned}
\phi\left(x_{n}, x_{0}\right) & =\phi\left(\Pi_{C_{n} x_{0}, x_{0}}\right) \\
& \leq \phi\left(\Pi_{\bigcap_{i \in \Lambda} F\left(T_{i}\right)} x_{0}, x_{0}\right)-\phi\left(\Pi_{\bigcap_{i \in \Lambda} F\left(T_{i}\right)} x_{0}, x_{n}\right) \\
& \leq \phi\left(\Pi_{\bigcap_{i \in \Lambda} F\left(T_{i}\right)} x_{0}, x_{0}\right) .
\end{aligned}
$$

This implies that the sequence $\left\{\phi\left(x_{n}, x_{0}\right)\right\}$ is bounded. It follows from (2.6) that the sequence $\left\{x_{n}\right\}$ is also bounded. Since $\left\{x_{n}\right\}$ is bounded and the space is uniformly convex, we may assume that $x_{n} \rightarrow \bar{x}$ weakly. Since $C_{n}$ is closed and convex, we see that $\bar{x} \in C_{n}$. On the other hand, we see from the weakly lower semicontinuity of the norm

$$
\begin{aligned}
\phi\left(\bar{x}, x_{0}\right) & =\|\bar{x}\|^{2}-2\left\langle\bar{x}, J x_{0}\right\rangle+\left\|x_{0}\right\|^{2} \\
& \leq \liminf _{n \rightarrow \infty}\left(\left\|x_{n}\right\|^{2}-2\left\langle x_{n}, J x_{0}\right\rangle+\left\|x_{0}\right\|^{2}\right) \\
& =\liminf _{n \rightarrow \infty} \phi\left(x_{n}, x_{0}\right) \\
& \leq \limsup _{n \rightarrow \infty} \phi\left(x_{n}, x_{0}\right) \\
& \leq \phi\left(\bar{x}, x_{0}\right),
\end{aligned}
$$

that $\phi\left(x_{n}, x_{0}\right) \rightarrow \phi\left(\bar{x}, x_{0}\right)$ as $n \rightarrow \infty$. Hence, $\left\|x_{n}\right\| \rightarrow\|\bar{x}\|$ as $n \rightarrow \infty$. In view of the KadecKlee property of $E$, we obtain $x_{n} \rightarrow \bar{x}$ as $n \rightarrow \infty$. On the other hand, we see from $x_{n+1}=$ $\Pi_{C_{n+1}} x_{0} \in C_{n+1} \subset C_{n}$ that

$$
\phi\left(x_{n}, T_{i}^{n} x_{n}\right) \leq \frac{2}{1-\kappa_{i}}\left\langle x_{n}-x_{n+1}, J x_{n}-J T_{i}^{n} x_{n}\right\rangle+\mu_{(n, i)} \frac{M_{(n, i)}}{1-\kappa_{i}}+\frac{\xi_{(n, i)}}{1-\kappa_{i}}
$$

from which it follows that $\phi\left(x_{n}, T_{i}^{n} x_{n}\right) \rightarrow 0$ as $n \rightarrow \infty$. In view of Lemma 2.12, we arrive at

$$
\lim _{n \rightarrow \infty}\left\|T_{i}^{n} x_{n}-x_{n}\right\|=0
$$

Note that $x_{n} \rightarrow \bar{x}$ as $n \rightarrow \infty$. From

$$
\left\|T_{i}^{n} x_{n}-\bar{x}\right\| \leq\left\|T_{i}^{n} x_{n}-x_{n}\right\|+\left\|x_{n}-\bar{x}\right\|
$$

we obtain from (3.7) that

$$
\lim _{n \rightarrow \infty}\left\|T_{i}^{n} x_{n}-\bar{x}\right\|=0 .
$$


On the other hand, we have

$$
\left\|T_{i}^{n+1} x_{n}-\bar{x}\right\| \leq\left\|T_{i}^{n+1} x_{n}-T_{i}^{n} x_{n}\right\|+\left\|T_{i}^{n} x_{n}-\bar{x}\right\| .
$$

It follows from the uniformly asymptotic regularity of $T_{i}$ and (3.8) that

$$
\lim _{n \rightarrow \infty}\left\|T_{i}^{n+1} x_{n}-\bar{x}\right\|=0
$$

That is, $T_{i} T_{i}^{n} x_{n} \rightarrow \bar{x}$. From the closedness of $T_{i}$, we find $\bar{x}=T_{i} \bar{x}$ for each $i \in \Lambda$. This proves $\bar{x} \in \bigcap_{i \in \Lambda} F\left(T_{i}\right)$.

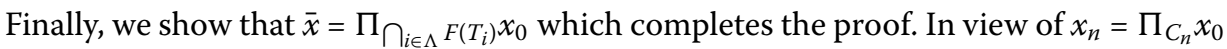
and $\Pi_{\bigcap_{i \in \Lambda} F\left(T_{i}\right)} x_{0} \in \bigcap_{i \in \Lambda} F\left(T_{i}\right) \subset C_{n}$, we obtain that

$$
\phi\left(x_{n}, x_{0}\right) \leq \phi\left(\Pi_{\bigcap_{i \in \Lambda} F\left(T_{i}\right)} x_{0}, x_{0}\right) .
$$

This implies that

$$
\phi\left(\bar{x}, x_{0}\right)=\lim _{n \rightarrow \infty} \phi\left(x_{n}, x_{0}\right) \leq \phi\left(\Pi_{\left.\bigcap_{i \in \Lambda} F\left(T_{i}\right) x_{0}, x_{0}\right)} .\right.
$$

This shows $\bar{x}=\Pi_{\bigcap_{i \in \Lambda} F\left(T_{i}\right) x_{0}}$. This completes the proof.

Remark 3.2 In view of spaces, algorithms, and mappings, Theorem 3.1 is a generalization of Theorem 4.1 of Sahu, Xu, and Yao [23]; see [23] for more details.

Based on Theorem 3.1, we have the following.

Corollary 3.3 Let E be a uniformly convex and smooth Banach space. Let $C$ be a nonempty closed and convex subset of $E$. Let $\Lambda$ be an index set and $T_{i}: C \rightarrow C$, where $i \in \Lambda$, be an asymptotically quasi- $\phi$-nonexpansive mapping in the intermediate sense. Assume that $\bigcap_{i \in \Lambda} F\left(T_{i}\right) \neq \emptyset$. For each $i \in \Lambda$, assume that $T_{i}$ is closed and uniformly asymptotically regular on $C$. Let $\left\{x_{n}\right\}$ be a sequence generated in the following manner:

$$
\left\{\begin{array}{l}
x_{0} \in E, \quad \text { chosen arbitrarily, } \\
C_{(1, i)}=C, \\
C_{1}=\bigcap_{i \in \Lambda} C_{(1, i)}, \\
x_{1}=\Pi_{C_{1}} x_{0}, \\
C_{(n+1, i)}=\left\{u \in C_{(n, i)}: \phi\left(x_{n}, T_{i}^{n} x_{n}\right) \leq 2\left\langle x_{n}-u, J x_{n}-J T_{i}^{n} x_{n}\right\rangle+\xi_{(n, i)}\right\}, \\
C_{n+1}=\bigcap_{i \in \Lambda} C_{(n+1, i)}, \\
x_{n+1}=\prod_{C_{n+1}} x_{0}, \quad \forall n \geq 0,
\end{array}\right.
$$

where

$$
\xi_{(n, i)}=\max \left\{0, \sup _{p \in F\left(T_{i}\right), x \in C}\left(\phi\left(p, T_{i}^{n} x\right)-\phi(p, x)\right)\right\} .
$$

Then the sequence $\left\{x_{n}\right\}$ converges strongly to $\bar{x}=\Pi_{\bigcap_{i \in \Lambda} F\left(T_{i}\right) x_{0}}$. 
Proof Putting $\mu_{n, i} \equiv 0$ and $\kappa_{i} \equiv 0$, we can conclude from Theorem 3.1 the desired conclusion immediately.

Remark 3.4 In view of algorithms and mappings, Corollary 3.3 is a generalization of Theorem 2.1 of Qin, Huang, and Wang [46]; see [46] for more details.

In the framework of Hilbert spaces, we have the following results for an uncountable family of asymptotically strict quasi-pseudocontractions in the intermediate sense and an uncountable family of asymptotically quasi-nonexpansive mappings in the intermediate sense.

Corollary 3.5 Let $H$ be a Hilbert space and $C$ a nonempty closed and convex subset of $H$. Let $\Lambda$ be an index set and $T_{i}: C \rightarrow C$, where $i \in \Lambda$, be an asymptotically strict quasipseudocontraction in the intermediate sense with a sequence $\left\{\mu_{(n, i)}\right\} \subset[0, \infty)$ such that $\mu_{(n, i)} \rightarrow 0$ as $n \rightarrow \infty$. Assume that $\bigcap_{i \in \Lambda} F\left(T_{i}\right) \neq \emptyset$. For each $i \in \Lambda$, assume that $T_{i}$ is closed and uniformly asymptotically regular on $C$, and $F\left(T_{i}\right)$ is bounded. Let $\left\{x_{n}\right\}$ be a sequence generated in the following manner:

$$
\left\{\begin{array}{l}
x_{0} \in H, \quad \text { chosen arbitrarily, } \\
C_{(1, i)}=C, \\
C_{1}=\bigcap_{i \in \Lambda} C_{(1, i)}, \\
x_{1}=P_{C_{1}} x_{0}, \\
C_{(n+1, i)}=\left\{u \in C_{(n, i)}:\right. \\
\left.\quad\left\|x_{n}-T_{i}^{n} x_{n}\right\|^{2} \leq 2\left\langle x_{n}-u, x_{n}-T_{i}^{n} x_{n}\right\rangle+\mu_{(n, i)} M_{(n, i)}+\xi_{(n, i)}\right\}, \\
C_{n+1}=\bigcap_{i \in \Lambda} C_{(n+1, i)}, \\
x_{n+1}=P_{C_{n+1}} x_{0}, \quad \forall n \geq 0,
\end{array}\right.
$$

where $M_{(n, i)}=\sup \left\{\phi\left(p, x_{n}\right): p \in F\left(T_{i}\right)\right\}$ and

$$
\xi_{(n, i)}=\max \left\{0, \sup _{p \in F\left(T_{i}\right), x \in C}\left(\left\|p-T_{i}^{n} x\right\|^{2}-\left(1+\mu_{(n, i)}\right)\|p-x\|^{2}-\kappa_{i}\left\|x-T_{i}^{n} x\right\|^{2}\right)\right\} .
$$

Then the sequence $\left\{x_{n}\right\}$ converges strongly to $\bar{x}=P_{\bigcap_{i \in \Lambda} F\left(T_{i}\right)} x_{0}$.

Corollary 3.6 Let $H$ be a Hilbert space and $C$ a nonempty closed and convex subset of $H$. Let $\Lambda$ be an index set and $T_{i}: C \rightarrow C$, where $i \in \Lambda$, be an asymptotically quasinonexpansive mapping in the intermediate sense. Assume that $\bigcap_{i \in \Lambda} F\left(T_{i}\right) \neq \emptyset$. For each $i \in \Lambda$, assume that $T_{i}$ is closed and uniformly asymptotically regular on $C$. Let $\left\{x_{n}\right\}$ be a 
sequence generated in the following manner:

$$
\left\{\begin{array}{l}
x_{0} \in H, \quad \text { chosen arbitrarily, } \\
C_{(1, i)}=C, \\
C_{1}=\bigcap_{i \in \Lambda} C_{(1, i)}, \\
x_{1}=P_{C_{1}} x_{0}, \\
C_{(n+1, i)}=\left\{u \in C_{(n, i)}:\left\|x_{n}-T_{i}^{n} x_{n}\right\|^{2} \leq 2\left\langle x_{n}-u, x_{n}-T_{i}^{n} x_{n}\right\rangle+\xi_{(n, i)}\right\}, \\
C_{n+1}=\bigcap_{i \in \Lambda} C_{(n+1, i)}, \\
x_{n+1}=P_{C_{n+1}} x_{0}, \quad \forall n \geq 0,
\end{array}\right.
$$

where

$$
\xi_{(n, i)}=\max \left\{0, \sup _{p \in F\left(T_{i}\right), x \in C}\left(\left\|p-T_{i}^{n} x\right\|^{2}-\|p-x\|^{2}\right)\right\} .
$$

Then the sequence $\left\{x_{n}\right\}$ converges strongly to $\bar{x}=P_{\bigcap_{i \in \Lambda} F\left(T_{i}\right)} x_{0}$.

\section{Applications}

In this section, we consider minimizers of proper, lower semicontinuous, and convex functionals, and solutions of variational inequalities.

Let $E$ be a Banach space with the dual $E^{*}$. For a proper lower semicontinuous convex function $f: E \rightarrow(-\infty, \infty]$, the subdifferential mapping $\partial f \subset E \times E^{*}$ of $f$ is defined by

$$
\partial f(x)=\left\{x^{*} \in E^{*}: f(x)+\left\langle y-x, x^{*}\right\rangle \leq f(y), \forall y \in E\right\}, \quad \forall x \in E .
$$

Rockafellar [47] proved that $\partial f$ is a maximal monotone operator. It is easy to verify that $0 \in \partial f(v)$ if and only if $f(v)=\min _{x \in E} f(x)$.

Theorem 4.1 Let E be a uniformly convex and smooth Banach space. Let $C$ be a nonempty, closed, and convex subset of $E$. Let $\Lambda$ be an index set, and $f_{i}: C \rightarrow C$ be a proper, lower semicontinuous, and convex functionals, for every $i \in \Lambda$. Assume that $\bigcap_{i \in \Lambda}\left(\partial f_{i}\right)^{-1}(0)$ is nonempty. Let $\left\{x_{n}\right\}$ be a sequence generated in the following manner:

$$
\left\{\begin{array}{l}
x_{0} \in E, \quad \text { chosen arbitrarily, } \\
C_{(1, i)}=C, \\
C_{1}=\bigcap_{i \in \Lambda} C_{(1, i)}, \\
x_{1}=\prod_{C_{1}} x_{0}, \\
y_{(n, i)}=\arg \min _{z \in E}\left\{f_{i}(z)+\frac{\|z\|^{2}}{2 r_{i}}+\frac{\left\langle z, J x_{n}\right\rangle}{2 r_{i}}\right\}, \\
C_{(n+1, i)}=\left\{u \in C_{(n, i)}: \phi\left(x_{n}, y_{(n, i)}\right) \leq 2\left\langle x_{n}-u, J x_{n}-J y_{(n, i)}\right\rangle\right\}, \\
C_{n+1}=\bigcap_{i \in \Lambda} C_{(n+1, i)}, \\
x_{n+1}=\prod_{C_{n+1}} x_{0}, \quad \forall n \geq 0,
\end{array}\right.
$$

where $r_{i}>0, \forall i \in \Lambda$. Then $\left\{x_{n}\right\}$ converges strongly to $\Pi_{\left.\bigcap_{i \in \Lambda}\left(\partial f_{i}\right)^{-1}(0)\right)_{0}}$, where $\Pi_{\bigcap_{i \in \Lambda}\left(\partial f_{i}\right)^{-1}(0)}$ stands for the generalized projection from $E$ onto $\bigcap_{i \in \Lambda}\left(\partial f_{i}\right)^{-1}(0)$. 
Proof For each $r_{i}>0$, and $x \in E$, we see that there exists a unique $x_{r_{i}} \in D\left(\partial f_{i}\right)$ such that $J x \in J x_{r_{i}}+r_{i} \partial f_{i}\left(x_{r_{i}}\right)$, where $x_{r_{i}}=\left(J+r_{i} \partial f_{i}\right)^{-1} J x$. Notice that

$$
y_{(n, i)}=\arg \min _{z \in E}\left\{f_{i}(z)+\frac{\|z\|^{2}}{2 r_{i}}+\frac{\left\langle z, J x_{n}\right\rangle}{r_{i}}\right\}
$$

is equivalent to

$$
0 \in \partial\left(f_{i}+\frac{\|\cdot\|^{2}}{2 r_{i}}+\frac{J x_{n}}{r_{i}}\right) y_{(n, i)}=\partial f_{i}\left(y_{(n, i)}\right)+\frac{J y_{(n, i)}}{r_{i}}+\frac{J x_{n}}{r_{i}} .
$$

This shows that $y_{n, i}=\left(J+r_{i} \partial f_{i}\right)^{-1} J x_{n}$. In view of Example 2.3 in Qin, Cho, and Kang [36], we find that $\left(J+r_{i} \partial f_{i}\right)^{-1} J$ is closed quasi- $\phi$-nonexpansive with $F\left(\left(J+r_{i} \partial f_{i}\right)^{-1} J\right)=\left(\partial f_{i}\right)^{-1}(0)$. Notice that every quasi- $\phi$-nonexpansive mapping is an asymptotically strict quasi- $\phi$ pseudocontraction in the intermediate sense. Following the proof of Theorem 3.1, we can immediately conclude the desired conclusion. This completes the proof.

Let $C$ be a nonempty, closed, and convex subset of a Banach space $E$. Let $A: C \rightarrow E^{\prime \prime}$ be a single valued monotone operator which is hemicontinuous; that is, continuous along each line segment in $C$ with respect to the weak" topology of $E^{*}$. Consider the following variational inequality problem of finding a point $x \in C$ such that

$$
\langle y-x, A x\rangle \geq 0, \quad \forall y \in C .
$$

In this chapter, we use $\operatorname{VI}(C, A)$ to denote the solution set of the variational inequality involving $A$. The symbol $N_{C}(x)$ stands for the normal cone for $C$ at a point $x \in C$; that is,

$$
N_{C}(x)=\left\{x^{\prime \prime} \in E^{*}:\left\langle y-x, x^{*}\right\rangle \leq 0, \forall y \in C\right\} .
$$

Theorem 4.2 Let E be a uniformly convex and smooth Banach space. Let $C$ be a nonempty, closed, and convex subset of $E$. Let $\Lambda$ be an index set, and $A_{i}: C \rightarrow E^{*}$ a single valued, monotone, and hemicontinuous operator. Assume that $\bigcap_{i \in \Lambda} V I\left(C, A_{i}\right)$ is not empty. Let $\left\{x_{n}\right\}$ be a sequence generated in the following manner:

$$
\left\{\begin{array}{l}
x_{0} \in E, \quad \text { chosen arbitrarily, } \\
C_{(1, i)}=C, \\
C_{1}=\bigcap_{i \in \Lambda} C_{(1, i)}, \\
x_{1}=\prod_{C_{1}} x_{0}, \\
y_{(n, i)}=V I\left(C, A_{i}+\frac{1}{r_{i}}\left(J-J x_{n}\right)\right), \\
C_{(n+1, i)}=\left\{u \in C_{(n, i)}: \phi\left(x_{n}, y_{(n, i)}\right) \leq 2\left\langle x_{n}-u, J x_{n}-J y_{(n, i)}\right\rangle\right\}, \\
C_{n+1}=\bigcap_{i \in \Lambda} C_{(n+1, i)}, \\
x_{n+1}=\prod_{C_{n+1}} x_{0}, \quad \forall n \geq 0,
\end{array}\right.
$$

where $r_{i}>0, \forall i \in \Lambda$. Then $\left\{x_{n}\right\}$ converges strongly to $\Pi_{\bigcap_{i \in \Lambda} V I\left(C, A_{i}\right)} x_{0}$, where $\Pi_{\bigcap_{i \in \Lambda} V I\left(C, A_{i}\right)}$ stands for the generalized projection from $E$ onto $\bigcap_{i \in \Lambda} V I\left(C, A_{i}\right)$. 
Proof Define a mapping $T_{i} \subset E \times E^{*}$ by

$$
T_{i} x= \begin{cases}A_{i} x+N_{C} x, & x \in C, \\ \emptyset, & x \notin C .\end{cases}
$$

By Rockafellar [48], we know that $T_{i}$ is maximal monotone, and $T_{i}^{-1}(0)=V I\left(C, A_{i}\right)$. For each $r_{i}>0$, and $x \in E$, we see that there exists a unique $x_{r_{i}} \in D\left(T_{i}\right)$ such that $J x \in J x_{r_{i}}+$ $r_{i} T_{i}\left(x_{r_{i}}\right)$, where $x_{r_{i}}=\left(J+r_{i} T_{i}\right)^{-1} J x$. Notice that

$$
y_{(n, i)}=V I\left(C, A_{i}+\frac{1}{r_{i}}\left(J-J x_{n}\right)\right),
$$

which is equivalent to

$$
\left\langle y-y_{n, i}, A_{i} y_{(n, i)}+\frac{1}{r_{i}}\left(J y_{(n, i)}-J x_{n}\right)\right\rangle \geq 0, \quad \forall y \in C,
$$

that is,

$$
-A_{i} y_{(n, i)}+\frac{1}{r_{i}}\left(J x_{n}-J y_{(n, i)}\right) \in N_{C}\left(y_{(n, i)}\right)
$$

This implies that $y_{(n, i)}=\left(J+r_{i} T_{i}\right)^{-1} J x_{n}$. In view of Example 2.3 in Qin, Cho, and Kang [31], we find that $\left(J+r_{i} \partial f_{i}\right)^{-1} J$ is closed quasi- $\phi$-nonexpansive with $F\left(\left(J+r_{i} \partial f_{i}\right)^{-1} J\right)=T_{i}^{-1}(0)$. Notice that every quasi- $\phi$-nonexpansive mapping is an asymptotically strict quasi- $\phi$ pseudocontraction in the intermediate sense. Following the proof of Theorem 3.1, we can immediately conclude the desired conclusion.

\section{Competing interests}

The authors declare that they have no competing interests.

\section{Authors' contributions}

All authors contributed equally to this manuscript. All authors read and approved the final manuscript.

\section{Author details}

'Department of Mathematics, Hangzhou Normal University, Hangzhou, 310036, China. ${ }^{2}$ College of Statistics and Mathematics, Yunnan University of Finance and Economics, Kunming, 650221, China. ${ }^{3}$ Department of Mathematics and RINS, Gyeongsang National University, Jinju, 660-701, Korea.

\section{Acknowledgements}

This research is partially supported by Natural Science Foundation of Zhejiang Province (Q12A010097) and National Natural Science Foundation of China (11126334)

Received: 16 April 2012 Accepted: 14 August 2012 Published: 7 September 2012

\section{References}

1. Bauschke, HH, Borwein, JM: On projection algorithms for solving convex feasibility problems. SIAM Rev. 38, 367-426 (1996)

2. Kotzer, T, Cohen, N, Shamir, J: Image restoration by a novel method of parallel projection onto constraint sets. Optim. Lett. 20, 1772-1774 (1995)

3. Byrne, $\mathrm{C}$ : A unified treatment of some iterative algorithms in signal processing and image reconstruction. Inverse Probl. 20, 103-120 (2008)

4. Censor, Y, Elfving, T, Kopf, N, Bortfeld, T: The multiple-sets split feasibility problem and its applications for inverse problems. Inverse Probl. 21, 2071-2084 (2005)

5. Lau, ATM, Miyake, H, Takahashi, W: Approximation of fixed points for amenable semigroups of nonexpansive mappings in Banach spaces. Nonlinear Anal. 67, 1211-1225 (2007) 
6. Kadelburg, Z, Radenovic, S: Coupled fixed point results under tvs-cone metric and w-cone-distance. Adv. Fixed Point Theory 2, 29-46 (2012)

7. Reich, S: Weak convergence theorems for nonexpansive mappings in Banach spaces. J. Math. Anal. Appl. 67, 274-276 (1979)

8. Schu, J: Weak, strong convergence to fixed points of asymptotically nonexpansive mappings. Bull. Aust. Math. Soc. 43, 153-159 (1991)

9. Khan, MA, Yannelis, NC: Equilibrium Theory in Infinite Dimensional Spaces. Springer, New York (1991)

10. Combettes, PL: The convex feasibility problem in image recovery. In: Hawkes, P (ed.) Advances in Imaging and Electron Physics, vol. 95, pp. 155-270. Academic Press, New York (1996)

11. Dautray, R, Lions, JL: Mathematical Analysis and Numerical Methods for Science and Technology, vols. 1-6. Springer, New York (1988-1993)

12. Fattorini, HO: Infinite-Dimensional Optimization and Control Theory. Cambridge University Press, Cambridge (1999)

13. Güler, O: On the convergence of the proximal point algorithm for convex minimization. SIAM J. Control Optim. 29, 403-409 (1991)

14. Haugazeau, Y: Sur les inéquations variationnelles et la minimisation de fonctionnelles convexes. Ph.D. Thesis, Université de Paris (1968)

15. Goebel, K, Kirk, WA: A fixed point theorem for asymptotically nonexpansive mappings. Proc. Am. Math. Soc. 35 171-174 (1972)

16. Bruck, RE, Kuczumow, T, Reich, S: Convergence of iterates of asymptotically nonexpansive mappings in Banach spaces with the uniform Opial property. Colloq. Math. 65, 169-179 (1993)

17. Chidume, CE, Shahzad, N, Zegeye, H: Convergence theorems for mappings which are asymptotically nonexpansive in the intermediate sense. Numer. Funct. Anal. Optim. 25, 239-257 (2004)

18. Kim, GE, Kim, TH: Mann and Ishikawa iterations with errors for non-Lipschitzian mappings in Banach spaces. Comput. Math. Appl. 42, 1565-1570 (2001)

19. Browder, FE, Petryshyn, WV: Construction of fixed points of nonlinear mappings in Hilbert space. J. Math. Anal. Appl. 20, 197-228 (1967)

20. Marino, G, Xu, HK: Weak and strong convergence theorems for strict pseudo-contractions in Hilbert spaces. J. Math. Anal. Appl. 329, 336-346 (2007)

21. Qihou, L: Convergence theorems of the sequence of iterates for asymptotically demicontractive and hemicontractive mappings. Nonlinear Anal. 26, 1835-1842 (1996)

22. Kim, TH, Xu, HK: Convergence of the modified Mann's iteration method for asymptotically strict pseudo-contractions. Nonlinear Anal. 68, 2828-2836 (2008)

23. Sahu, DR, Xu, HK, Yao, JC: Asymptotically strict pseudocontractive mappings in the intermediate sense. Nonlinear Anal. 70, 3502-3511 (2009)

24. Alber, YI: Metric and generalized projection operators in Banach spaces: properties and applications. In: Kartsatos, AG (ed.) Theory and Applications of Nonlinear Operators of Accretive and Monotone Type. Dekker, New York (1996)

25. Cioranescu, I: Geometry of Banach Spaces, Duality Mappings and Nonlinear Problems. Kluwer, Dordrecht (1990)

26. Hudzik, H, Kowalewski, W, Lewicki, G: Approximative compactness and full rotundity in Musielak-Orlicz spaces and Lorentz-Orlicz spaces. Zeitschrift fuer Analysis and ihre Anwendungen 25, 163-192 (2006)

27. Takahashi, W: Nonlinear Functional Analysis. Yokohama-Publishers, Yokohama (2000)

28. Reich, S: A weak convergence theorem for the alternating method with Bregman distance. In: Karstsatos, AG (ed.) Theory and Applications of Nonlinear Operators of Accretive and Monotone Type. Dekker, New York (1996)

29. Butnariu, D, Reich, S, Zaslavski, AJ: Asymptotic behavior of relatively nonexpansive operators in Banach spaces. J. Appl. Anal. 7, 151-174 (2001)

30. Butnariu, D, Reich, S, Zaslavski, AJ: Weak convergence of orbits of nonlinear operators in reflexive Banach spaces. Numer. Funct. Anal. Optim. 24, 489-508 (2003)

31. Censor, Y, Reich, S: Iterations of paracontractions and firmly nonexpansive operators with applications to feasibility and optimization. Optimization 37, 323-339 (1996)

32. Su, Y, Qin, X: Strong convergence of modified Ishikawa iterations for nonlinear mappings. Proc. Indian Acad. Sci. Math. Sci. 117, 97-107 (2007)

33. Agarwal, RP, Cho, YJ, Qin, X: Generalized projection algorithms for nonlinear operators. Numer. Funct. Anal. Optim. 28, 1197-1215 (2007)

34. Qin, X, Su, Y, Wu, C, Liu, K: Strong convergence theorems for nonlinear operators in Banach spaces. Commun. Appl. Nonlinear Anal. 14, 35-50 (2007)

35. Qin, X, Cho, YJ, Kang, SM: Convergence theorems of common elements for equilibrium problems and fixed point problems in Banach spaces. J. Comput. Appl. Math. 225, 20-30 (2009)

36. Zhou, H, Gao, G, Tan, B: Convergence theorems of a modified hybrid algorithm for a family of quasi- $\phi$-asymptotically nonexpansive mappings. J. Appl. Math. Comput. 32, 453-464 (2010)

37. Qin, X, Cho, YJ, Kang, SM, Zhu, H: Convergence of a modified Halpern-type iteration algorithm for quasi- $\boldsymbol{\phi}$-nonexpansive mappings. Appl. Math. Lett. 22, 1051-1055 (2009)

38. Qin, X, Cho, SY, Kang, SM: On hybrid projection methods for asymptotically quasi- $\boldsymbol{\phi}$-nonexpansive mappings. Appl. Math. Comput. 215, 3874-3883 (2010)

39. Qin, X, Cho, SY, Kang, SM: Strong convergence of shrinking projection methods for quasi- $\phi$-nonexpansive mappings and equilibrium problems. J. Comput. Appl. Math. 234, 750-760 (2010)

40. Qin, X, Agarwal, RP: Shrinking projection methods for a pair of asymptotically quasi- $\boldsymbol{\phi}$-nonexpansive mappings. Numer. Funct. Anal. Optim. 31, 1072-1089 (2010)

41. Ye, J, Huang, J: Strong convergence theorems for fixed point problems and generalized equilibrium problems of three relatively quasi-nonexpansive mappings in Banach spaces. J. Math. Comput. Sci. 1, 1-18 (2011)

42. Qin, X, Agarwal, RP, Cho, SY, Kang, SM: Convergence of algorithms for fixed points of generalized asymptotically quasi- $\phi$-nonexpansive mappings with applications. Fixed Point Theory Appl. 2012, 58 (2012)

43. Qin, X, Wang, T, Cho, SY: Hybrid projection algorithms for asymptotically strict quasi- $\phi$-pseudocontractions. Abst. Appl. Anal. 2011, Article ID 142626 (2011) 
44. Zhou, H, Gao, X: An iterative method of fixed points for closed and quasi-strict pseudo-contractions in Banach spaces. J. Appl. Math. Comput. 33, 227-237 (2010)

45. Kamimura, S, Takahashi, W: Strong convergence of a proximal-type algorithm in a Banach space. SIAM J. Optim. 13 938-945 (2002)

46. Qin, X, Huang, S, Wang, T: On the convergence of hybrid projection algorithms for asymptotically quasi- $\boldsymbol{\phi}$-nonexpansive mappings. Comput. Math. Appl. 61, 851-859 (2011)

47. Rockafellar, RT: Characterization of the subdifferentials of convex functions. Pac. J. Math. 17, 497-510 (1966)

48. Rockafellar, TT: On the maximality of sums of nonlinear monotone operators. Trans. Am. Math. Soc. 149, 75-88 (1970)

doi:10.1186/1687-1812-2012-143

Cite this article as: Qin et al.: Some results on fixed points of asymptotically strict quasi- $\phi$-pseudocontractions in the intermediate sense. Fixed Point Theory and Applications 2012 2012:143.

Submit your manuscript to a SpringerOpen ${ }^{\odot}$ journal and benefit from:

- Convenient online submission

- Rigorous peer review

- Immediate publication on acceptance

- Open access: articles freely available online

- High visibility within the field

- Retaining the copyright to your article

Submit your next manuscript at $>$ springeropen.com 